\section{Frühes NLPHL nur bestrahlen?}

Das noduläre lymphozytenprädominante Hodgkin-Lymphom (NLPHL) ist relativ selten, die optimale Therapie im frühen Stadium unklar. Die deutsche Hodgkin-Studiengruppe analysierte gepoolte Daten aus verschiedenen Studien, um die derzeit optimale Therapie herauszufinden.

E s standen Daten von 256 Patienten - mit NLPHL des Stadiums IA zur Verfügung, die zwischen 1988 und 2009 mit unterschiedlichen Studienprotokollen behandelt worden waren. 72 Patienten hatten kombinierte Therapiemodalitä-

ten (CMT) mit unterschiedlichen Chemotherapie- und Bestrahlungsprotokollen erhalten, 49 eine erweiterte Bestrahlung („extended-field radiotherapy“, EFRT), 108 eine Involved-Field-Strahlentherapie (IF-RT) und 27 Patienten eine

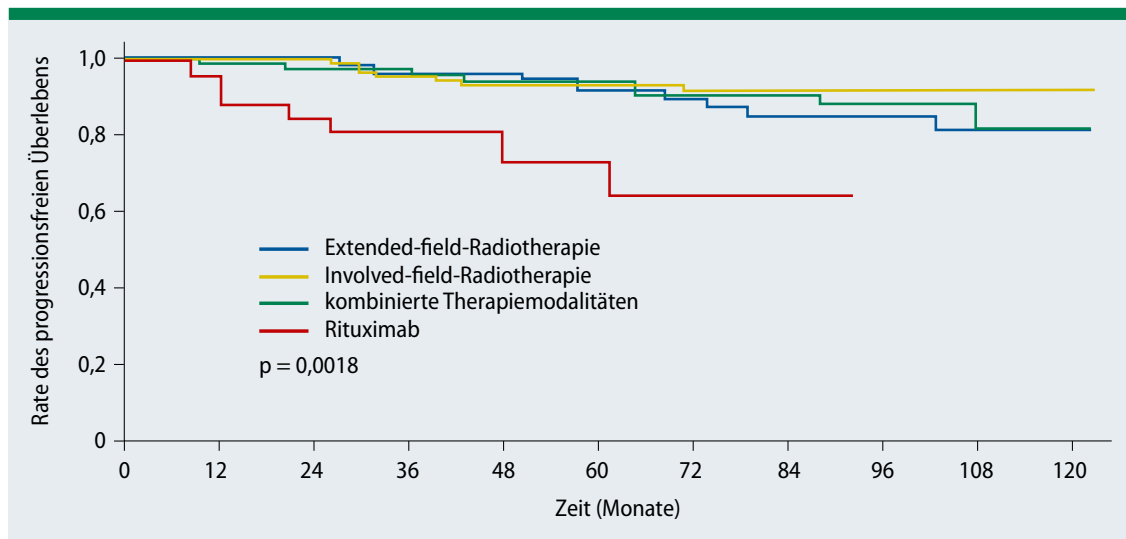

Abb. 1: Eine Rituximab-Monotherapie ist allen anderen Optionen hinsichtlich des Progressionsrisikos unterlegen.

\section{Morbus Hodgkin: PET-geführte Therapiewahl}

Bei Hodgkin-Lymphomen (HL) im Stadium III oder IV gibt es zwei unterschiedlich toxische Therapieregimes. In einer US-Studie wurde der Wechsel auf die toxischere Behandlung bei positivem PET/CT-Ergebnis geprüft.

A Is Erstlinien-Standard beim fortgeschrittenen HL gilt in den USA ABVD (Doxorubicin, Bleomycin, Vinblastin, Dacarbacin), in Deutschland BEACOPP $_{\text {eskaliert }}$ (Bleomycin, Etoposid, Doxorubicin, Cyclophosphamid, Vincristin, Procarbazin, Prednison), das effektiver, aber toxischer ist. Im Jahr 2014 fand sich nur bei 15-45\% der Patienten, die nach 2 Zyklen ABVD noch PET(Positronenemissionstomografie)-positiv waren, ein langfristiges progressionsfreies Überleben (PFS). Deshalb wurde in einer US-Studie geprüft, ob sich bei PET/CT (Computertomografie)-positiven Patien- ten nach 2 Zyklen ABVD das Therapieergebnis durch den Wechsel auf BEA$\mathrm{COPP}_{\text {eskaliert }}$ verbessern lässt. 358 Patienten im Stadium III (52\%) oder IV (48\%) erhielten zunächst 2 Zyklen ABVD. Die folgende PET-Untersuchung war bei 60 Patienten positiv. Von 55 für 6 Zyklen BEACOPP $_{\text {eskaliert }}$ vorgesehenen Patienten wurden 49 so behandelt. In der PET-negativen Vergleichsgruppe erhielten 270 Patienten 4 weitere ABVD-Zyklen.

In letzterer Gruppe erzielten $96 \%$ der Patienten eine Komplettremission, in der Gruppe mit Therapiewechsel bei positivem PET/CT sprachen $55 \%$ komplett 4-wöchige Therapie mit Rituximab $\left(375 \mathrm{mg} / \mathrm{m}^{2}\right)$. Im Median wurde die gesamte Studienpopulation 91 Monate nachbeobachtet.

Die Raten für progressionsfreies Überleben und Gesamtüberleben nach 8 Jahren betrugen bei CMT 88,5 und $98,6 \%$, bei EF-RT 84,3 und $95,7 \%$ und bei IF-RT 91,9 und 99,0\%. Für Patienten mit die Rituximab-Monotherapie lagen die entsprechenden Raten nach 4 Jahren bei 81,0 und $100 \% .17$ von 256 Patienten $(6,6 \%)$ entwickelten ein Zweitmalignom.

Fazit: Das progressionsfreie Überleben und damit die Tumorkontrolle scheinen dieser Analyse zufolge bei CMT, EF-RT und IF-RT vergleichbar. Wegen des relativ niedrigen Toxizitätsrisikos sollte die IF-RT den Wissenschaftlern zufolge als Therapiestandard bei einem NLPHL im Stadium IA betrachtet werden. Eine alleinige Rituximab-Therapie ist nach diesen Analysen mit einem höheren Progressionsrisiko assoziiert als die übrigen Optionen (Abb. 1).

Friederike Klein

Eichenauer DA et al. Long-Term Course of Patients With Stage IA Nodular Lymphocyte-Predominant Hodgkin Lymphoma: A Report From the German Hodgkin Study Group. J Clin Oncol. 2015;33(26):2857-62.

und $38 \%$ partiell an. Die geschätzte PFSRate nach 2 Jahren lag in der PET-negativen Gruppe bei $82 \%$, in der Gruppe mit positivem PET und Therapiewechsel bei $64 \%$, d.h. wesentlich höher als in früheren Studien mit $30 \%$. Wie erwartet war BEACOPP eskaliert $_{\text {toxischer als ABVD }}$ (Grad-4/5-Toxizitäten: 85,7 vs. 36,7\%).

Fazit: Durch eine Therapieintensivierung mit $\mathrm{BEACOPP}_{\text {eskaliert }}$ - orientiert am Ergebnis von PET/CT-Untersuchungen - lässt sich bei anfangs mit ABVD behandelten HL-Patienten ein längeres PFS erzielen als mit ABVD - jedoch bei höherer Toxizität. Peter Leiner

Press OW et al. US Intergroup Trial of Response-Adapted Therapy for Stage III to IV Hodgkin Lymphoma Using Early Interim Fluorodeoxyglucose-Positron Emission Tomography Imaging: Southwest Oncology Group S0816. J Clin Oncol. 2016 Apr 11. [Epub ahead of print]. 